\title{
REMOTE HEALTH MONITORING SYSTEM USING HEALTHY PI
}

\section{BRAJAKISHOREBARIK ${ }^{1}$, DEBRAJARANA ${ }^{2}$ \& SANTANUMOHAPATRA ${ }^{3}$}

${ }^{I}$ M-Tech Scholar, Centurion University of Technology and Management, Bhubaneswar, Odisha, India

${ }^{2}$ Assistant Professor, Department of Electronics and Communication Engineering, Centurion

University of Technology and Management, Bhubaneswar, Odisha, India

${ }^{3}$ Team Lead, Infosys, Bhubaneswar, Odisha, India

\begin{abstract}
Remote health monitoring system can be implemented to monitor the health status of the patient by the doctor to avoid critical situations through an alarm. The proposed system presents a personal healthcare system that is both flexible and scalable. This model incorporates Healthy Pi V3 kit, three sensor modules to measure body temperature, heartbeat rate, respiratory rate and ECG along with two transmitting systems. One transmitting system Sends sensor outputs through Intranet which will help doctors/ caretakers to be alert and other system sends these sensors outputs through web service to doctor's or relative's mobile phone using Android and Windows Applications. This smart health monitoring system is a real-time embedded system can be used at remote places, where internet speed is very slow. This is very useful for future analysis and review of the patient's health condition. This model can be further improvised, by incorporating blood pressure monitoring systems, dental sensors etc. to make it more useful in hospitals as a very efficient and dedicated patient monitoring system.

KEYWORD: Healthy Pi connections: Windows App \& Android App
\end{abstract}

Received: Mar 28, 2017; Accepted: Apr 17, 2018; Published: Apr 24, 2018; Paper Id: IJEEERJUN20181

\section{INTRODUCTION}

The Smart Remote health monitoring system is an extension of a hospital medical system where a patient's vital body state can be monitored remotely by Android or Windows systems. IOT has its lots of contribution in the medical field as a latest example is BSN technology. It provides the environment, where vital parameters get transmitted by medical devices like body sensors to a public cloud and secured private cloud in which information can be stored, assembled and analyzed. This way such type of cloud can store millions of patient's health-related data which can be future analyzed.

\section{Objective of Design}

Now a day's Healthcare system can be analyzed and discovered with respect to the application areas in medical practice. Some of the applications areas are as follows along with the usage of IOT concept and their benefits to humans.

\section{- Wireless Monitoring System for Patients}

This system is essential for identifying patient's essential parameters and activities remotely with the help of sensors situated on the human body. In this model, the real-time data is being shared and send to the caretakers and doctors. This system is specially used for the management of disease like heart disease, 
hypertension, diabetes, asthma, etc. where the patient needs to be continuously monitored.

\section{- Access of Mobile Devices}

Access to Mobile/Laptop devices is completely based on the technologies that allow hospitalization systems to be remotely or virtually accessed. All these hospitalization systems can be continuously monitored using an Android/Windows application.

\section{- On-Line Consultation}

On-line consultation is based on the remotely consulting the patient with the help of multimedia system. It also helps in educating people about their health, medicine delivery and consultation in the case of emergency. Due to the consultation time is getting too longer in many countrie's, people have to face many problems. On-line consultation of a patient can be done in few seconds. The remote problem detecting with the help of video conferencing has become common. It is also called Tele-consultations.

\section{Benefits}

\section{Cost Effective for Patient}

Here the patient can be monitored from a far distance. So, it minimizes patient's traveling cost, hospital bill and time wastage for multiple visits.

\section{Fast Services}

This system provides immediate service to the patient by the doctor at any time, any place and anywhere.

\section{Disease Management on Real Time Basis}

Here the patient is get treated as early as possible before their health gets too much worse.

\section{Improvement of Life Quality}

This system helps aged people and also critically ill people to improve their life quality due to continuous monitoring by the health experts.

\section{PROPOSED SYSTEM}

In Modern Healthcare System, Internet of things is used to give flexibility and fast operational speed to get expected outcomes. My system hardware incorporates Raspberry pi 3, ECG sensor, Temperature sensor and SpO2 sensor etc. and more sensors also can be used to detect various biological functionality. These Hardware elements are embedded with a software system that controls the hardware and displays report. By using This System, we can detect abnormal conditions in the human body at a real-time.

Raspberry pi3 is connected with sensors, sensors are connected with human bodies, and this raspberry pi3 is connected with software systems by using Internet/Intranet connection. When all elements are connected together, sensor senses data from the human body, then sends that data to a server. After that these data are compared with standard values that are already stored in the system. The outcomes after comparison can check the normal and abnormal condition of a patient. If there are any critical situation occurring then the system sends a message immediately along with sensor outputs to doctors/healthcare exports for getting advice. A System Administrator is used to control all these above-said functions 
and also updates the database time to time with a few sec delays for new patient's entries and doctor's entries. In this system, we required two servers, one for system deployment and another for the database, which stores data. Since all the data is in a Digital format so it gives advantage on operational speed. For connectivity, I have made two separate arrangements, one using Intranet system and other using Internet system. In both cases, I have used a concept of an alarm system to alert doctors/caretakers.

In Intranet connection system communication between doctor/caretaker and a patient can take place by using $\mathrm{Wi}$ Fi system and VNC viewer, which is an open source software Application along with an alarm system.

In Internet connection system I have employed a new concept of Windows Application as well as Android Application along with alarm system. The purpose of using this new concept is to receive high quality, more secure and fast processing data from both sides. For designing windows Application XML (eXtensible Markup Language) is used and for Android Application JSON (JavaScript Object Notation) is used. The details about these two Languages are described below.

Sensors used mainly low power and it gives an advantage for long life operationality. Data is coming from sensors to raspberry pi is processed to convert it to a digital format that's make easy to process data in the system so that it gives advantage on operational speed. Normally digital processing is much more efficient than analog signal processing. For connectivity, we are using the PuTTY application to connect devices and software system that works like a mediator. When raspberry pi sends data, it comes to PuTTY and then it forwards to database system through App. To develop my system, it is more preferred to use a paid server which is costlier and it can be manageable when more and more people will use my system. In case of the open source server, security and data handling are two major issues, which can't be compromised when other's life issue is concerned.

This System is made to avoid critical situations and treatment on time and immediately. When abnormal data is indicated, the message will be send to doctor's mobile. And it can avoid risk and handle the critical situation. It also gives an advantage that it reduces time laps between situation and their alert to a doctor, that means the doctor will know the situation as immediately it happens. In this system, we are using BSN care server to deploy system and also using a separate database server to store data. JSON/XML is used to display both graphical and textual format of sensor data. Separate tables are made to store data which is coming from sensors. Sensors are connected to (LPU) Local Processing Unit to Process data into digital format. All these components are able to connect with a system with wire media and wireless also, hence it gives high portability to operate from a remote place. It also gives centralize control to the system by an administrator. Administrator is one user that can control the whole system. 


\section{Complete Block Diagram of Proposed System}

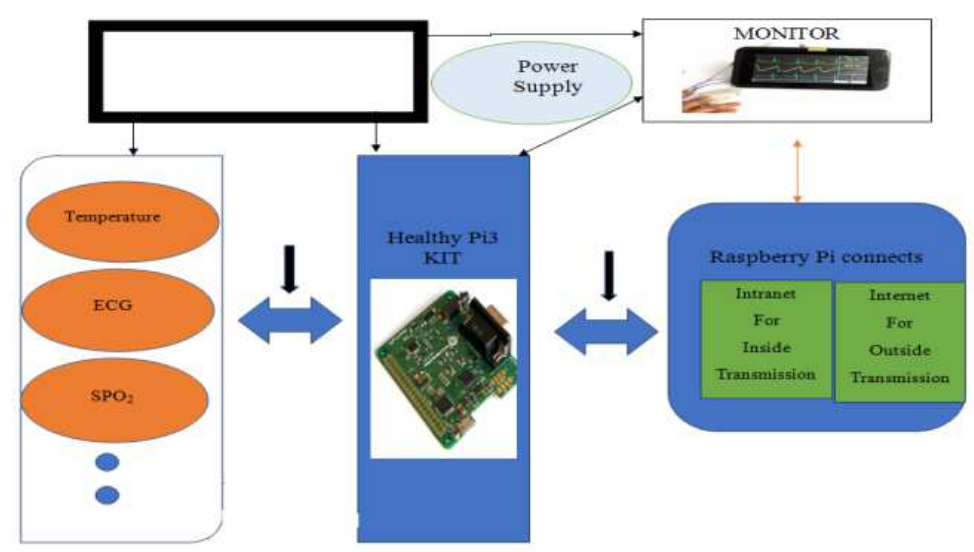

Figure 1: Propose System

The proposed system is the development of Smart Health Monitoring System which detects patient's health status continuously, like temperature, Respiratory Rate, ECG and SPO2etc and sends patient's vital information to doctor via SMS. My system also identifies patient's state (conscious, death, sleep) depending on their motion and updates alarms accordingly. System Maintain a complete log of patient activity automatically at doctor's end on a PC (A customized software shall be developed for the same). It creates audio-visual alarms at patients end for nurses or other people to respond and alert them. To captures, patient's image is a future analysis and need more work on that.

\section{Features}

- System can able to monitor heart rate, respiratory rate, temperature, etc. of multiple patients at a time.

- The system also sends the vital data (undershoot or overshoots in values) to the administrator via SMS by using Android/Windows Applications.

- Administrator can able to send the data/commands back to the system.

- $\quad$ Large data can be stored and easily accessed.

- A GUI based software graphically displays all the vital information in form of graphs.

- Administrator changes the threshold values of alarms for individual patients either via SMS or via direct controlling the GUI based application provided.

\section{Architecture of Healthy Pi-Connect}

Healthy Pi Connect is an android/windows application which shows the reports/data of healthy Pi on the computer or mobile devices. 


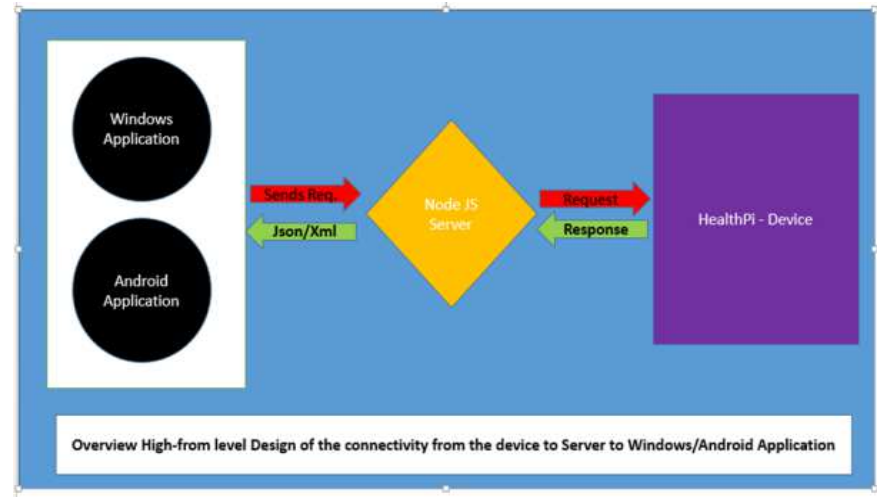

Figure 2: Raspberry Pi Connect

\section{Benefits of Healthy Pi-Connect}

- smart operations.

- Make our system more portable.

- $\quad$ More efficient.

- More secure

- $\quad$ More applicable.

\section{Sample Code of Healthy Pi-Connect}

- Configuration to Connect Serial Port/USB

<Devicecapability Name=" serialcomminication">

<Device Id=" any">

$<$ Function Type=" name:serialport" />

</Device $>$

$</$ DeviceCapability $>$

This code helps to connect server as well as the Raspberry Pi to connect directly.

\section{- Code to Design Calculations Result Output}

public sealed class StartupTask: IBackgroundTask

$$
\{
$$

While (true)$$
\{
$$

Vardevices=DeviceInformation.FindAllAsync(SerialDevice.GetDeviceSelector())

Devices.Wait();

foreach (vardev in devices.Result) 


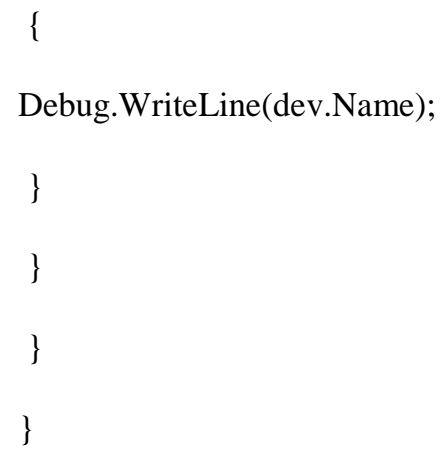

Sample Request and Response to Flow Data between Server and Mobile Device Signup

http://localhost:8080/healthpy/signUp

\section{Request}

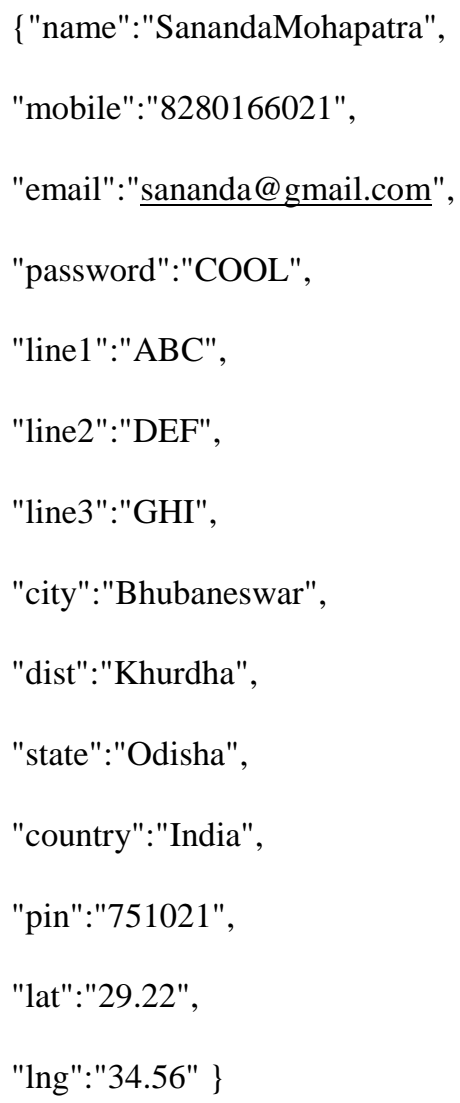

\section{Response}

"Status": \{ "statusCode": "REGISTRATION_PROCESS_COMPLETED",

"statusMessage": "Registration process completed."

\}

\} 


\section{$\operatorname{LogIn}$}

http://192.168.0.123:8080/healthy/signin

\section{Request}

\{

"password":"COOL",

"emailId":"ronychatterjee@gmail.com"

\}

\section{Response}

\{

"Status": \{

"statusCode": "CUSTOMER_LOGIN_VERIFIED",

"statusMessage": "Login Successful."

\},

"CustomerRegistrationDetails": \{

"customerId": 1,

"name": "SanandaMohapatra",

"mobileNumber": "8180166021",

"email": "sananda@gmail.com",

"role": 1 ,

"address": 1,

"password": "COOL",

"customerActiveStatus": 1,

"createdDate": "01/12/2018 17:29:47",

"createdBy": "SYSTEM",

"updatedDate": null,

"updatedBy": null,

"deviceId": null

\}

\} 


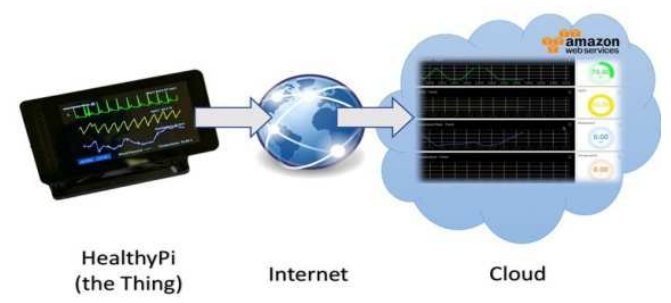

Figure 3: Example of a Healthy Pi Connection

\section{Healthy Pi Connect Through SSH}

- To SSH on windows, we will need an additional tool called Putty.

- Download and install the putty.exe.

- In this screen, we will need to input the IP of the Raspberry Pi into the Host Name Field.

- $\quad$ The port should be 22

- Now click on open

- Now it will be prompted with a security alert, simply click yes to this.

- Now login with the default username "pi" and password "raspberry".

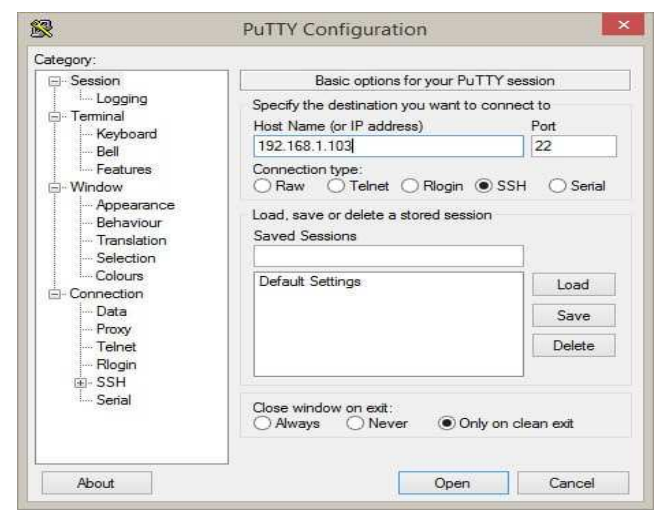

Figure 4: PuTTY Configuration

\section{Install Real VNC Server on Raspberry pi}

Take SSH Session of raspberry pi using Putty.

Install VNC Server from Command Line

- Before we get started first make sure Raspbian OS is up to date by running the following commands:

sudo apt-get update

sudo apt-get upgrade

- Run the following commands to make sure you have the latest version of Real VNC.

sudo apt-get install realvnc-vnc-server

sudo apt-get install realvnc-vnc-viewer 
- During Install, it will take default password as "raspberry"

\section{Install VNC Server Graphically}

- Before we get started first make sure Raspbian OS is up to date by running the following commands: sudo apt-get update sudo apt-get upgrade

- Run the following commands to get a graphical version of Raspberry PI Configuration utility. sudoraspi-config

- Navigate to Interfacing Options.

- $\quad$ Scroll down and select VNC > Yes

- During Install, it will take default password as "raspberry"

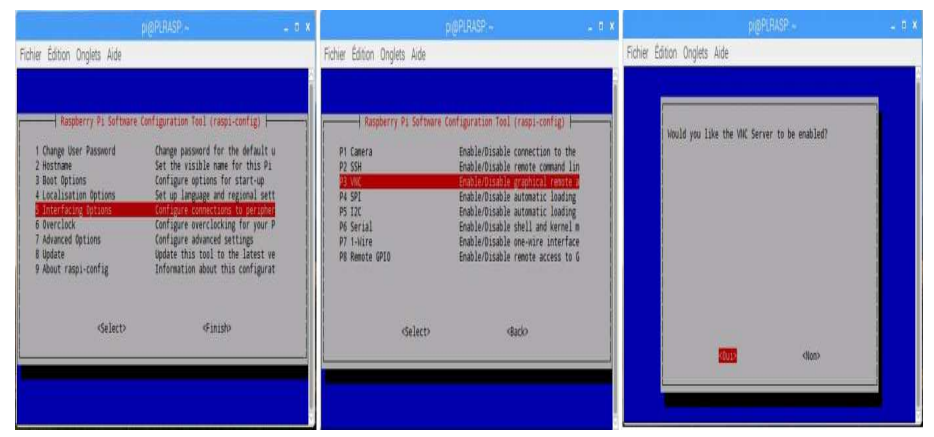

Figure 5

\section{Install Real VNC Viewer on Desktop or Mobile}

- Download Real VNC Viewer Client from its site.

- $\quad$ For Android mobile download from Google Play Store.

- Enter your IP address in the field that says VNC server.

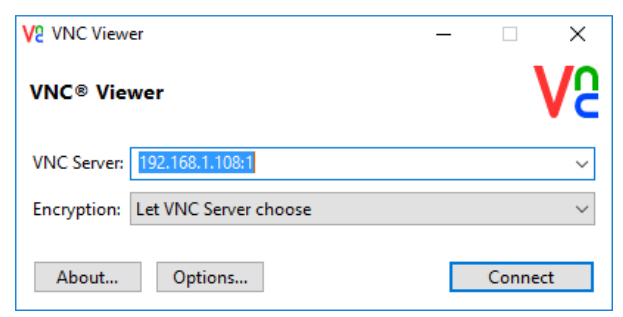

Figure 6

- Now simply press connect.

- It should then give you a warning message, simply press continue on this.

- Then it will ask you for the password you set for it earlier. Simply enter the password as "raspberry"

- $\quad$ The VNC window should load and you will now have access to your Raspberry Pi remotely. 


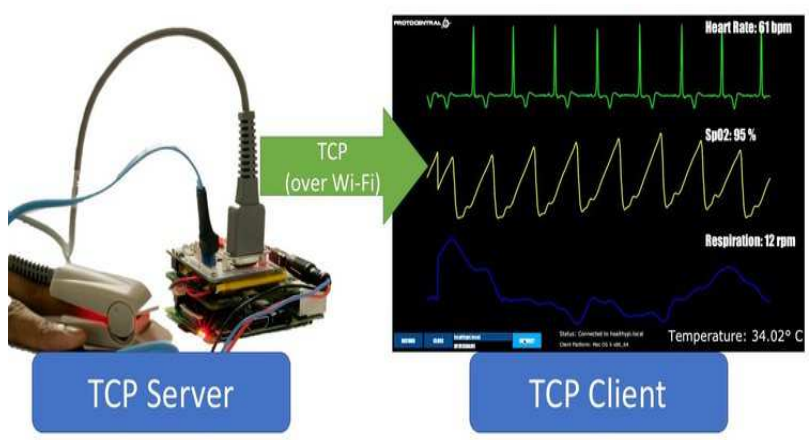

Figure 7

\section{OUTPUT}

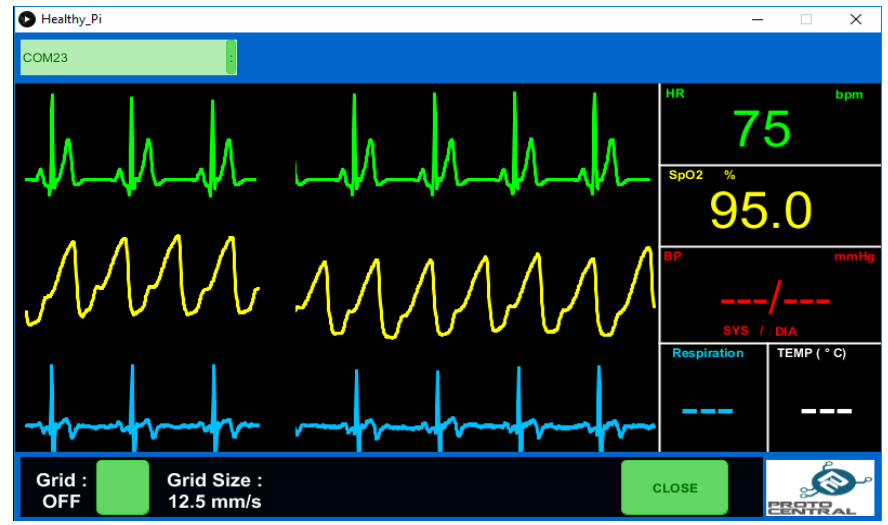

Figure 8

\section{SUMMARY OF THE STUDY}

The remote patient monitoring system was researched, designed and presented around the concept of the Internet of things. Personal physiological data from the patient is collected that simulates respiratory rate and the heartbeat. The readings are collected in a simple local database and can be viewed remotely by a doctor or Healthcare giver through Android and Windows Application. An alarm system is integrated into this system to alert doctors and caretakers for the emergency situation. The data can also be used in research on medical issues affecting the elderly or chronically ill.

On security of the data, the database system is protected Advanced Encryption Standard (AES). This generates the secret key which can be used to decrypt the patients' records ensuring that only authorized personnel access the data. This safeguards the patients' records from unauthorized users and hackers who may want to intercept.

\section{CONCLUSIONS AND FUTURE WORK}

This paper totally fulfills primary requirements like reliability, scalability, and range. My proposed system can detect patients body states continuously like temperature, ECG, BP etc. The Healthy Pi device and Android/Windows Apps both together are used for performing, information gathering, storing, and analysis. Security and privacy on all levels of the layer are also taking care of with respect to performance and comfortable use. This system can be further integrated to other surveillance systems for security and remote location management. In this paper, I have tried to put my views in details conceptually to design an IOT based 'Smart Remote Health Monitoring System' using body sensors to full fill all requirements in healthcare service. 


\section{SUGGESTION FOR FURTHER STUDY}

\section{- Physiological Data Collection}

Home Ultrasound

Brain signal monitoring

\section{- $\quad$ Remote viewing of data}

Data privacy/security especially of medical systems.

Mobile phones may be designed with all these facilities.

\section{ACKNOWLEDGEMENT}

I would like to thank God, for His unending grace and love throughout my academic life. Without it, I would not have made it this far.

I would also like to extend my sincere gratitude to my guide, Prof. DebrajaRana for his priceless motivation, guidance and support.

I would again like to thank my HOD Prof. Dr. S K Mandal Sir, Prof Deepak Ku Barik and all my lecturers and all non-teaching staffs for their contribution.

To my classmates, I am thankful for their moral support as I did the project and the conducive environment.

Finally, I would like to thank my family for their help and understanding throughout my academic life.

\section{REFERENCES}

1. International Journal of Computer Networks \& Communications (IJCNC) Vol.7, No.3, May 2015 DOI: 10.5121/ijcnc.2015.7302 13 REAL TIME WIRELESS HEALTH MONITORING APPLICATION USING MOBILE DEVICES Amna Abdullah, Asma Ismael, Aisha Rashid, Ali Abou-ElNour, and Mohammed Tarique Department of Electrical Engineering, Ajman University of Science and Technology, P. O. Box 2202, Fujairah, United Arab Emirates.]

2. A Real-Time Health Monitoring System for Remote Cardiac Patients Using Smartphone and Wearable Sensors $\underline{\text { Priyanka Kakria, }}^{1} \underline{\text { N. K. Tripathi }},{ }^{1}$ and Peerapong Kitipawang ${ }^{2}$

3. V. K. Chippa and S. T. Chakradhar and K. Roy, "Analysis and characterization of inherent application resilience for approximate computing”, in DAC, 2013.

4. Survey on secure communication protocols for the internet of things. Ad Hoc Networks (2015)

5. Ahmed, M. U., Banaee, H., Loutfi, A., Rafael-Palou, X.: Intelligent healthcare services to support health monitoring of elderly. In: HealthyIoT (2014)

6. FarzadSamie, Lars Bauer, Jörg Henkel, “IoT Technologies for Embedded Computing: A Survey”, ESWEEK'16, October 2-7 2016, Pittsburgh, PA, USA.

7. Alkadi, Bashir Thabit, and Alan Abubaker Ghafoor. "Derivation of Spatially Distributed Unit Hydrograph for Balisan Valley Watershed using Gis and Remote Sensing Techniques."

8. Ahmed, M. U., Espinosa, J. R., Reissner, A., Domingo, A., Banaee, H., Loutfi, A., `Rafael-Palou, X.: Self-serve ict-based health monitoring to support active ageing. In: HEALTHINF (2015) 
9. (C) 2014 IJEDR | Volume 2, Issue 1 | ISSN: 2321-9939 IJEDR1401108 International Journal of Engineering Development and Research (www.ijedr.org) 605 Advanced Health Monitoring and Receiving Using Smartphone in Global Networks 1 Jayalakshmi R, 2Mahalingam D, 3Rajeswari A Nandha Engineering College, Erode

10. Review”, 2016 IEEE $40^{\text {th }}$ Annual computer software and application conference, DOI 10.1109/COMPSAC.2016.8.

11. M. U. Ahmed, M. Bjorkman, and A. Cau`sevic, et al. An overview on the internet of things for health monitoring systems.In IoT Technologies for HealthCare, 2015.

12. F. Samie and L. Bauer and C.-M. Hsieh et al. Online binding of applications to multiple clock domains in shared fpgabased systems. In DATE, pp. 25-30, 2015.

13. C. Perera and A. Zaslavsky and P. Christen et al. Context aware computing for the Internet of Things: A survey. IEEE Communications Surveys \& Tutorials, 16(1):414-454, 2014.

\section{APPENDICES}

\section{Flow Chart}

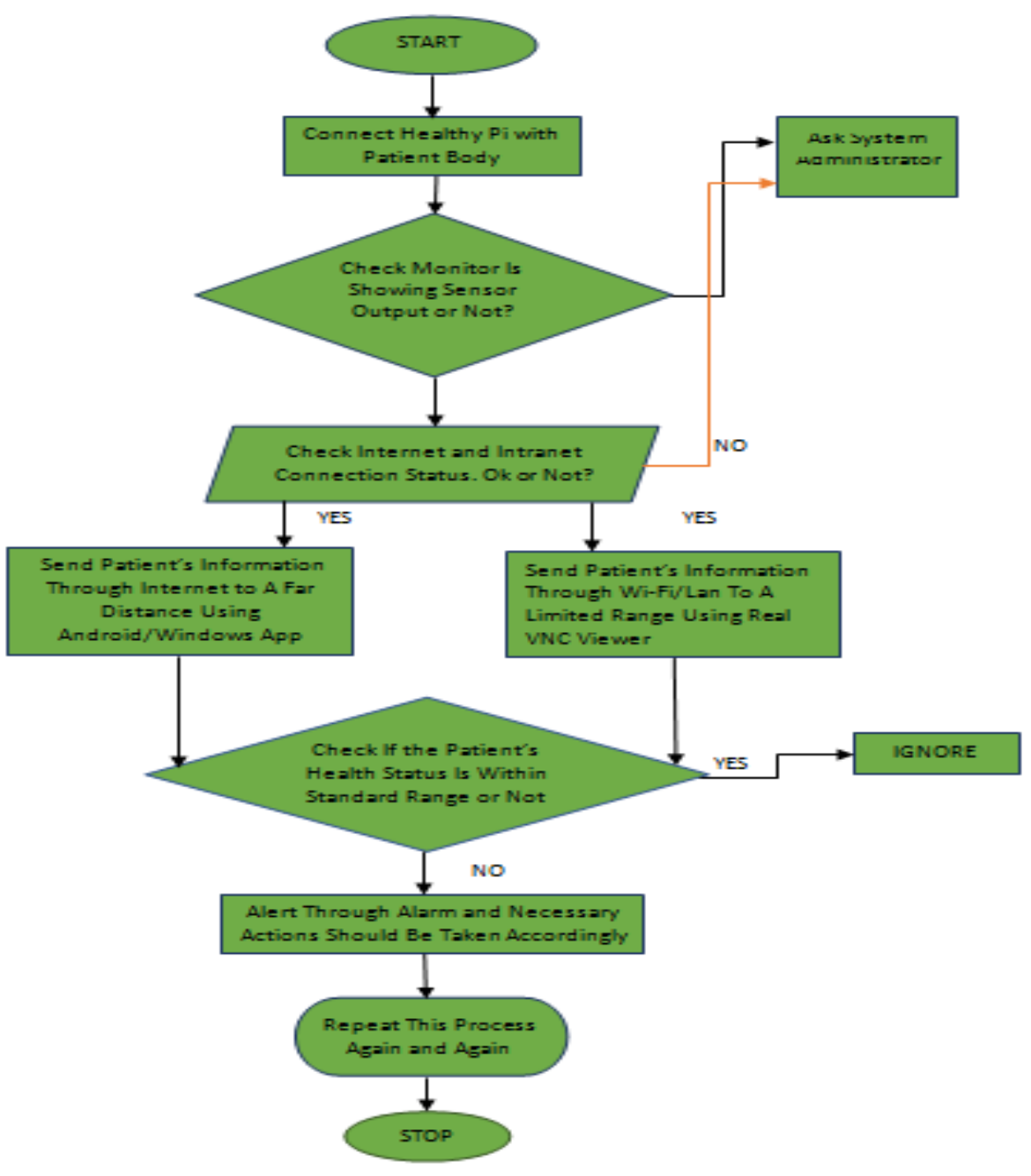

